\title{
ATTAIN: Phase III study of etirinotecan pegol versus treatment of physician's choice in patients with metastatic breast cancer and brain metastases
}

\author{
Debu Tripathy*,1, Sara M Tolaney², Andrew D Seidman³, Carey K Anders ${ }^{4}$, Nuhad Ibrahim, \\ Hope S Rugo ${ }^{5}$, Chris Twelves ${ }^{6,7}$, Veronique Dieras ${ }^{8}$, Volkmar Müller ${ }^{9}$, Mary Tagliaferri ${ }^{10}$, \\ Alison L Hannah ${ }^{10}$ \& Javier Cortés ${ }^{11,12}$ \\ ${ }^{1}$ The University of Texas MD Anderson Cancer Center, Department of Breast Medical Oncology, Houston, TX 77030, USA \\ ${ }^{2}$ Dana-Farber Cancer Institute, Center for Women's Cancers, Boston, MA 02215, USA \\ ${ }^{3}$ Memorial Sloan-Kettering Cancer Center, Bobst International Center, New York, NY 10065, USA \\ ${ }^{4}$ University of North Carolina School of Medicine, Duke Cancer Center, Chapel Hill, NC 27710, USA \\ ${ }^{5}$ University of California San Francisco, Department of Medicine (Hematology/Oncology), San Francisco, CA 94115, USA \\ ${ }^{6}$ University of Leeds, Leeds Institute of Cancer and Pathology (LICAP), Leeds, LS2 9JT, UK \\ ${ }^{7}$ St James' University Hospital, Institute of Oncology, Leeds, LS9 7BE, UK \\ ${ }^{8}$ Institut Curie, Oncological Medicine Department, 75248, Paris, France \\ ${ }^{9}$ University Medical Center Hamburg-Eppendorf, Department of Obstetrics and Gynecology, 20246 Hamburg, Germany \\ ${ }^{10}$ Nektar Therapeutics, San Francisco, CA 94158, USA \\ ${ }^{11}$ IOB Institute of Oncology, Quironsalud Group, 28034 Madrid \& 08023 Barcelona, Spain \\ ${ }^{12}$ Vall d'Hebron Institute of Oncology (VHIO), Breast Cancer and Melanoma Group, 08035 Barcelona, Spain \\ *Author for correspondence: Tel.: +1 (713) 792 2817; Fax: (713) 563 0903; dtripathy@mdanderson.org
}

The increasing incidence of breast cancer brain metastases is a major clinical problem with its associated poor prognosis and limited treatment options. The long-acting topoisomerase- 1 inhibitor, etirinotecan pegol, was designed to preferentially accumulate in tumor tissue including brain metastases, providing sustained cytotoxic SN38 levels. Motivated by improved survival findings from subgroup analyses from the Phase III BEACON trial, this ongoing randomized, Phase III trial compares etirinotecan pegol to drugs commonly used for advanced breast cancer in patients with stable, treated breast cancer brain metastases who have been previously treated with an anthracycline, taxane and capecitabine. The primary end point is overall survival. Secondary end points include objective response rate, progression-free survival and time to CNS disease progression or recurrence in patients with/without CNS lesions present at study entry. Trial registration number: NCT02915744.

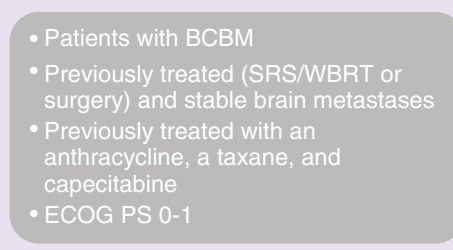

Stratification by:

- Geographic region

- Hormone and HER2 receptor status

(TNBC, HER2+, or HR+/HER2-)

- ECOG PS

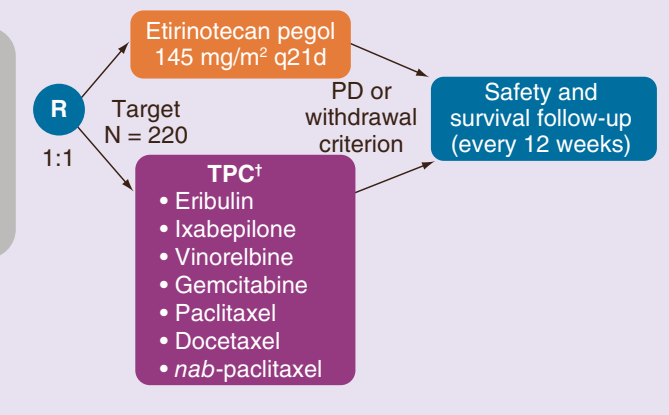

First draft submitted: 28 March 2019; Accepted for publication: 17 April 2019; Published online: 10 May 2019

Keywords: brain metastases $\bullet$ chemotherapy • etirinotecan pegol • metastatic breast cancer $\bullet$ NKTR-102 
The incidence of brain metastases (BM) among women with metastatic breast cancer (MBC) has been significantly underestimated, likely due to patients succumbing to systemic progression prior to symptomatic CNS disease [14]. In recent years, the incidence of $\mathrm{BM}$ has been estimated to be as high as $30 \%$ in unselected patients and approaching or exceeding 35-50\% in subpopulations with HER2-positive or 'triple-negative' (TNBC; estrogen and progesterone receptor negative, HER2 normal) disease [2,3,5-15]. This high incidence of BM is thought to be associated with prolonged survival secondary to improvements in the management of systemic disease and better imaging studies, but also in large part due to the lack of efficacy and CNS penetration of existing therapies [3$5,16,17]$. Despite advances in the management of $\mathrm{MBC}$, breast cancer brain metastases (BCBM) remains a significant clinical problem, portending a poor prognosis and leading to neurocognitive deterioration as well as interruptions in systemic disease management.

Treatment of BCBM is driven primarily by critical prognostic factors (age, tumor subtype, performance status, number of $\mathrm{BM}$ and presence of extracranial metastases) and comprises varying combinations of surgery $(+/-$ adjuvant radiotherapy to whole brain or tumor-bed alone), whole-brain radiation therapy (WBRT; $+/$ - a focal boost), stereotactic radiosurgery (SRS; alone or $+/-$ WBRT) and systemic therapies (chemotherapy or targeted therapy, either separately or in conjunction with other localized therapeutic approaches) [2-7,10-12,16,18-26]. The most widely used treatment for patients with multifocal BCBM is WBRT, most notably in patients with BM that are not amenable to surgical resection or in select populations, such as those with poor performance status or rapidly progressing systemic disease $[3,6,18]$. The evolution of SRS (alone or in combination) into a safe and focal high-dose treatment for BCBM has been demonstrated by consistent rates of local control in most series [23,27-31]. While improved intracranial control has been demonstrated, survival evidence is lacking and patients are at risk of serious quality-of-life altering adverse effects, including significant neurocognitive impairment and decline [3$6,11,16,18-21,23,26,30]$. Careful patient selection is key as timing and appropriate use of these modalities remains debated.

Chemotherapy has traditionally played a limited role in the management of BCBM due to formidable obstacles, including the blood-brain barrier (BBB), with its tight junctions that limit the blood-to-brain passage of many agents $[10,16,32]$. Additionally, despite the apparent structural and functional breakdown of the BBB secondary to $\mathrm{BM}$ as well as RT, and the subsequent development of new permeable vasculature (blood-tumor barrier or BTB), data indicate that the BBB and BTB remain sufficiently intact to significantly impair drug delivery (although trastuzumab emtansine, a large monoclonal antibody drug conjugate, has been shown in case reports to result in intracranial responses in selected patients) [33-36]. Furthermore, the BBB and BTB harbor various efflux transporters, including P-glycoprotein and breast cancer resistance protein (BCRP), which bind many of the chemotherapeutics approved for MBC and render them ineffective [32].

It is typically desired that BCBM be treated with systemic chemotherapy before or after RT or surgery, and more recently as an appealing option to avoid or delay WBRT and its associated complications. However, there are no approved chemotherapy regimens indicated for the management of BCBM, nor are there consensusbased recommendations for the general chemotherapeutic management with reasonable level of evidence, except for a guideline on the disease management of patients with HER2-positive breast cancer and BM published in $2014[3,5,6,10,12,22,37,38]$. Although SRS may be repeatedly administered for untreated and, in some instances, for radiated lesions, treatment options are limited for patients with BCBM that relapse or progress after initial surgery/RT/SRS therapeutic approaches, supported by small prospective trials reporting only modest response rates and short durations of benefit $[3,7,10,11,22,38,39]$. Targeted agents alone or with chemotherapy are being explored; however, the results have been conflicting in this heavily pretreated population [40-44]. Cross-resistance, the primary impediment to the successful treatment of patients in the advanced disease setting, as well as overlapping and/or cumulative toxicities, most commonly neurotoxicity and myelosuppression, also frequently preclude the use of commonly used agents in the BCBM setting $[11,45-47]$. Thus, novel systemic treatment approaches are critically needed.

\section{Etirinotecan pegol (NKTR-102)}

Etirinotecan pegol (EP; also known as NKTR-102) is a novel long-acting topoisomerase I inhibitor-polymer conjugate engineered by attaching irinotecan molecules via a biodegradable cleavable ester-based linker to a polyethylene glycol (PEG) polymer core. Through in vivo hydrolysis of the cleavable linker, EP was specifically 
designed to provide a slow and extended release of irinotecan and subsequently slower transformation to its main active metabolite, SN38 (7-ethyl-10-hydroxycamptothecin), resulting in reduced maximum plasma concentrations (C-max) yet sustained SN38 concentrations [48]. Preclinical and early clinical trials demonstrated an approximate tenfold reduction in the C-max of SN38 after EP administration compared with administration of irinotecan and a prolongation of the SN38 half-life to approximately 50 days, which is in contrast to the half-life of SN38 following conventional administration of irinotecan of $\leq 2$ days [48-51]. By not overwhelming the capacity of the carboxyl-esterase enzymes that deacetylate irinotecan to SN38, the slow release of irinotecan from EP allows for a more efficient drug activation and resultant greater SN38 area under the concentration time-curve (AUC). This continuous exposure to $\mathrm{SN} 38$ is intended to improve antitumor activity while providing a more favorable toxicity profile.

In addition to the pharmacokinetic (PK) advantages, the EP polymer conjugate achieves selective drug targeting by exploiting abnormalities of the tumor vasculature, a phenomenon called the enhanced permeability and retention effect or EPR [48,52,53]. This unique phenomenon of solid tumors is based on their anatomical and pathophysiological differences from normal tissues, namely hypervascularization, aberrant architecture, hyperpermeability and lack of lymphatic drainage. Because EP is a large macromolecule, it penetrates the leaky vasculature within the tumor environment more readily than normal vasculature, concentrating and trapping the drug in the tumor tissue providing prolonged exposure of the tumor.

\section{The ATTAIN study}

Here we describe the design and rationale for ATTAIN (NCT02915744), an ongoing open-label, randomized, multicenter, international Phase III study of EP versus treatment of physician's choice (TPC) in patients with MBC and stable BM. The study is funded by Nektar Therapeutics, San Francisco, CA, USA.

\section{Background \& rationale for EP in the treatment of BCBM \\ Preclinical \& early clinical investigations}

Currently, available chemotherapeutic agents distribute poorly to lesions in the brain due to difficulties penetrating the BBB and BTB, and, because many of these chemotherapeutics are substrates of the efflux transporters expressed at these sites $[32,33]$. EP is not a substrate of such efflux transporters, and as demonstrated in early clinical investigations, tumor localization via EPR is pronounced and sustained, likely from the prolonged circulation in plasma, resulting in high concentrations of SN38 in brain tumors [48,54,55]. In a mouse xenograft BM model, EP exhibited preferential accumulation and generated higher SN38 concentrations in brain tissue over corresponding plasma concentrations (170- and 30-fold, respectively) [54,55]. Analyses of brain sections assessing for drug uptake demonstrated that the SN38 concentration in brain tissue was 100-fold greater and more prolonged subsequent to EP administration compared with non-conjugated irinotecan administration. This prolonged SN38 brain exposure was associated with a significant regression of established BM (size and number) and a doubling of the animals' median survival (74 days vs 37 days, for EP and irinotecan, respectively). The efficacy observed in this preclinical model demonstrates the ability of EP to cross the BTB, leading to preferential accumulation and retention in brain tumor, followed by sustained and efficacious concentrations of SN38.

Additional evidence for activity in the CNS was reported from a Phase II study in patients with bevacizumabrefractory, high-grade glioma [56]. In this open-label study, 18 patients (with a median of three prior therapies) were diagnosed with glioblastoma multiforme; three of the 18 (17\%) had a partial response to single-agent EP, with two responses lasting for $\geq 18$ months. Additionally, a Phase II trial for patients with BCBM testing iniparib and irinotecan yielded a response rate of $12 \%$, primarily attributed to irinotecan [57]. These data further support the preclinical evidence for the activity of irinotecan-based drugs within the brain.

\section{Phase III BEACON trial results}

The BrEAst Cancer Outcomes with NKTR-102 (BEACON; NCT01492101) trial was a Phase III, open-label, randomized, multicenter study of EP versus TPC in patients with heavily pretreated, locally recurrent or MBC previously treated with an anthracycline, a taxane and capecitabine [58]. Eligible patients had received a minimum of two prior cytotoxic regimens for advanced disease and no more than five for breast cancer in any setting. The study was designed to compare the overall survival (OS) of patients treated with EP given every 3 weeks $(\mathrm{n}=429)$ or a single-agent TPC $(n=423)$, a control arm that allowed investigators to choose one of seven commonly used cytotoxic drugs (eribulin, ixabepilone, vinorelbine, gemcitabine, paclitaxel, docetaxel or nab-paclitaxel) administered 


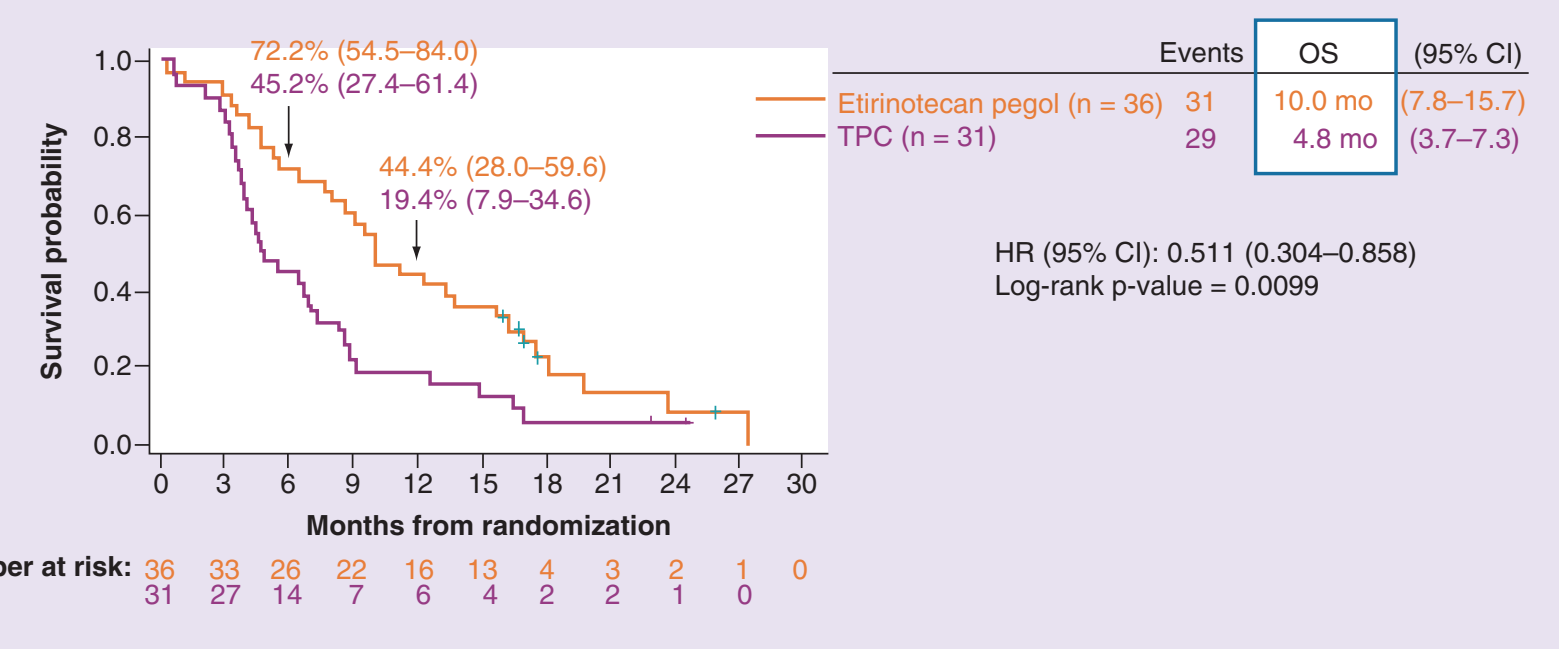

Figure 1. Kaplan-Meier plot of overall survival for patients with stable, treated brain metastases in the BEACON Phase III trial. EP: Etirinotecan pegol; HR: Hazard ratio; OS: Overall survival; TPC: Treatment of physician's choice. Created using data from Shah et al. [54].

according to local practice. Based on the preclinical data suggesting benefit, patients with a history of stable treated $\mathrm{BM}$ were eligible for inclusion and were specified as a subgroup of interest in the analysis plan prior to the final analysis of the trial. Ninety percent of the patients enrolled in the BEACON study had HER2-negative disease. Patients in both treatment groups received a median of three treatment cycles. In the overall study population, EP was associated with a 2.1-month median OS benefit over TPC (12.4 vs 10.3 months; $\mathrm{p}=0.084$ ); the hazard ratio (HR) of 0.87 (95\% CI: 0.747-1.019) missed statistical significance. However, EP was associated with statistically significant survival improvements $(\mathrm{p}<0.05)$ relative to TPC in important, prospectively defined subgroups with poor prognoses, including patients with liver metastases at baseline (median OS: 10.9 vs 8.3 months, respectively; HR: 0.73) and those with stable, pretreated BM at study entry as described below.

\section{BM subgroup analysis}

A total of 67 patients with a history of BM were randomized in the BEACON study. Eligibility criteria required previous treatment with surgical resection, WBRT and/or stereotactic radiation, use of corticosteroids for BM discontinued for $\geq 3$ weeks prior to randomization, and that signs or symptoms of BM were stable for $\geq 28$ days prior to randomization. No known progression of BM (as assessed by Response Evaluation Criteria in Solid Tumors [RECIST]) was permitted, and patients with leptomeningeal disease or meningeal carcinomatosis were excluded. In the primary survival analysis, 60 deaths occurred in the BCBM subgroup (EP: 31 [86\%]; TPC: 29 [94\%]). The prespecified BCBM subgroup analyses demonstrated a significant improvement in survival with a doubling of OS for patients receiving EP (10 months) versus TPC (4.8 months) [59]. This reduction in death resulted in a HR of 0.51 (95\% CI: 0.304-0.858; $<<0.01$ ) (Figure 1). The median OS of 4.8 months for patients with BCBM treated with TPC was consistent with reported median OS from previously published clinical studies in this setting, with OS ranges of 5.0 to 6.4 months [39]. EP was also associated with improvements in 6-and 12-month survival rates; corresponding to 72.2 and $45.2 \%$ for EP and TPC, respectively, and 44.4 and $19.4 \%$, respectively (Figure 1). Median progression-free survival (PFS) was 3.1 months for EP and 2.7 months for TPC (HR: 0.840; 95\% CI: $0.492-1.433 ; p=0.52$ ). In patients with measurable systemic disease, partial responses were observed in five of 32 (15.6\%) patients treated with EP and 1 of 27 (3.7\%) patients on TPC; there were no complete responses in either treatment group.

The survival trends favoring EP over TPC were also observed (post hoc) in 37 patients with radiographic evidence of brain lesions at baseline. Median OS for this subgroup was 13.2 months (95\% CI: 8.6-19.6) for EP ( $\mathrm{n}=19)$ and 5.8 months (95\% CI: 3.5-8.6) for TPC $(\mathrm{n}=18)$. In order to correct for potential imbalances between the groups, the BEACON investigators undertook a post hoc evaluation utilizing the Graded Prognostic Assessment (GPA) Index, an assessment tool that assigns scores for significant prognostic indices of Karnofsky performance 


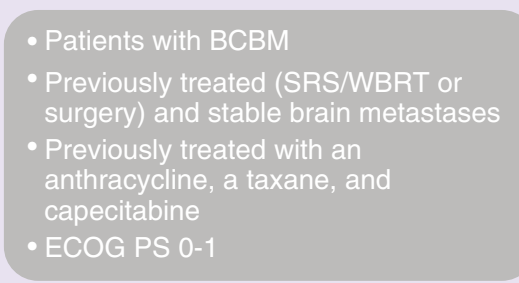

\section{Stratification by:}

- Geographic region

- Hormone and HER2 receptor status

(TNBC, HER2+, or HR+/HER2-)

- ECOG PS
Etirinotecan pegol

$145 \mathrm{mg} / \mathrm{m}^{2}$ q21d

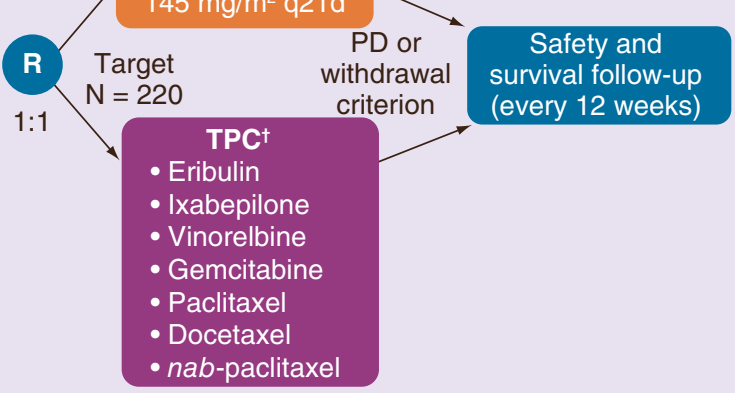

Figure 2. Study design.

$\dagger$ The agent must be available at the treating institution.

BCBM: Breast cancer brain metastases; ECOG: Eastern Cooperative Oncology Group; HR: Hormone receptor; PD: Progressive disease; PS: Performance status; q21d: Every 21 days; SRS: Stereotactic radiosurgery; TNBC: Triple-negative breast cancer; TPC: Treatment of physician's choice; WBRT: Whole-brain radiation therapy.

score (KPS), tumor subtype and age that was developed to predict prognosis in patients with newly diagnosed BM [24,25]. In these analyses, treatment with EP was associated with improved OS for patients in both better and worse prognosis groups; the HR for OS of the 67 patients after adjusting for the two GPA groups was 0.467 favoring EP, reinforcing the activity of EP in BCBM patients.

\section{Safety of EP}

The nature, scope and severity of the safety findings to date with EP have been clinically manageable and consistent. In the BEACON trial [58,60] and the BCBM subgroup [59], EP demonstrated a significantly lower rate of grade 3 or greater and dose-limiting/quality of life-reducing toxicities (notably neuropathy, myelosuppression and alopecia). EP was associated with more gastrointestinal toxicities, including diarrhea, with grade 3 diarrhea reported as 9.6 versus $1 \%$ in the entire BEACON population and 5.9 versus $3.7 \%$ in the BCBM subgroup for EP and TPC, respectively; no grade 4 diarrhea was reported.

In the analysis of patient-reported outcomes in the BEACON trial [61], there was evidence of benefit associated with EP compared with current standard of care agents in multiple Health-Related Quality of Life measurements, including global health status and physical functioning, despite worse gastrointestinal symptoms (e.g., diarrhea). Differences were seen favoring EP up to 32 weeks for global health status (GHS) and physical functioning scales $(\mathrm{p}<0.02)$; numerical improvement was reported in other functional scales.

The encouraging survival findings, the favorable safety profile and quality of life findings among the BEACON study patients with BCBM together with the Phase II evidence of single-agent activity in recurrent high-grade primary brain tumors, provides strong rationale and justification for the further exploration of EP in patients with BCBM previously treated with an anthracycline, taxane and capecitabine.

\section{ATTAIN study design}

\section{Study design \& objectives}

ATTAIN will randomize up to 220 patients to EP $\left(145 \mathrm{mg} / \mathrm{m}^{2}\right.$ every 21 days [q21d] as a 90 -min intravenous [iv.] infusion on day 1 of each treatment cycle) or to TPC consisting of single-agent iv. chemotherapy limited to one of the following seven agents: eribulin, ixabepilone, vinorelbine, gemcitabine, paclitaxel, docetaxel or nabpaclitaxel (administered [every 21 to 28 days] per standard of care) (Figure 2). Treatment will continue until disease progression per RECIST version 1.1 [62] and/or Response Assessment in Neuro-Oncology Brain Metastases (RANO-BM) criteria [63], unacceptable toxicity, patient withdrawal, investigator decision, loss to follow-up, death, noncompliance, pregnancy or study termination by the sponsor. Crossover to EP is not allowed.

The investigator must determine at screening which TPC regimen will be offered to the patient as part of the informed consent process and provide that choice to the independent contract research organization responsible 
Box 1. ATTAIN trial objectives.

Primary objective

- To compare OS of patients who receive EP with OS of patients who receive TPC selected from the following list of seven single-agent intravenous therapies: eribulin, ixabepilone, vinorelbine, gemcitabine, paclitaxel, docetaxel or nab-paclitaxel

Secondary objectives

- To compare the ORR between the two treatments; assessment of tumor outside the CNS will use

- RECIST (version 1.1) criteria; assessment of CNS metastases will use RANO-BM criteria

- To compare PFS of the two treatments; assessment of tumor outside the CNS will use RECIST (version 1.1) criteria; assessment of CNS metastases will use RANO-BM

- To compare the CBR of the two treatments (i.e., the proportion of patients having CR, PR, or SD for at least 4 months); CBR for peripheral lesions and for CNS lesions will be separately described

- To compare DoR of the two treatments; peripheral lesions and CNS lesions will be separately described

- To compare the time to CNS disease progression in those patients with CNS lesions present at study entry

- To compare the time to CNS recurrence in those patients with without CNS lesions present at study entry

- To compare the incidence and cumulative frequency of WBRT after date of randomization

- To compare the time-to-WBRT in those patients who received WBRT after the date of randomization

- To evaluate the safety profiles of EP and TPC (eribulin, ixabepilone, vinorelbine, gemcitabine, paclitaxel, docetaxel, or nab-paclitaxel)

- To compare HRQoL between the two treatments using the EORTC QLQ C30 with the BN-20 questionnaire, EQ $5 \mathrm{D}-5 \mathrm{~L}^{\mathrm{TM}}$ and the BFI

- To evaluate PK data (in patients randomized to EP only)

- To correlate presence of reduced function UGT1A1 variants with EP safety (in patients randomized to EP only)

- To evaluate the pharmacoeconomic implications of EP therapy using selected measures of health care utilization

- To evaluate the magnitude of clinical benefit using ESMO-MCBS

Exploratory objectives

- To identify biomarkers that correlate with ORR, PFS and OS

BFI: Brief Fatigue Inventory: BN-20: Brain neoplasms 20-question subscale; CBR: Clinical benefit rate; CR: Complete response; DoR: Duration of response; EORTC QLQ C30: European Organisation for Research and Treatment of Cancer Quality of Life Core 30 questionnaire; EP: Etirinotecan pegol; EQ 5D-5LTM: EuroQol 5D questionnaire; ESMO-MCBS: European Society for Medical Oncology magnitude of clinical benefit scale; HRQoL: Health-related quality of life; ORR: Objective response rate; OS: Overall survival; PK: Pharmacokinetic; PR: Partial response; PFS: Progression-free survival; RANO-BM: Response Assessment in Neuro-Oncology Brain Metastases; RECIST: Response Evaluation Criteria in Solid Tumors; SD: Stable disease; TPC: Treatment of physician's choice; WBRT: Whole-brain radiation therapy.

for creating and administering the randomization scheme. Patients will then be randomized centrally (1:1) via an interactive response system to EP or the registered TPC regimen for the individual patient. Patients will be stratified by geographic region (US vs rest of world), tumor receptor status (TNBC versus HER2-positive versus hormone receptor-positive/HER2-negative) and Eastern Cooperative Oncology Group (ECOG) performance status (0 vs $1)$.

The primary objective is to compare OS between the two treatment groups. Secondary and exploratory objectives are listed in Box 1 and include objective response rate [outside the CNS (ORR) and in BM (ORR-BM)], PFS (outside the CNS, in BM [PFS-BM] and CNS plus peripheral [PFS-overall]), clinical benefit rate (CBR), duration of response (DoR), time to CNS progression in patients with CNS lesions present at study entry, time to CNS recurrence in patients without CNS lesions present at study entry, incidence and cumulative frequency of WBRT, time-to-WBRT in those patients who received WBRT after randomization, PK, correlation of reduced function UGT1A1 variants with EP safety, pharmacoeconomic implications, toxicity and health-related quality of life (HRQoL).

\section{Eligibility criteria}

Key eligibility criteria are listed in Supplementary Table 1 and include patients willing and able to comply with the protocol and provide written informed consent prior to study-specific screening procedures, are female or male, age $\geq 18$ years, and have histologically confirmed carcinoma of the breast (either primary or metastatic lesion) for which single-agent cytotoxic chemotherapy is indicated. Patients may have either measurable or non-measurable metastatic disease according to RECIST, version 1.1 but must have a history of BM that are non-progressing and that had been previously treated with either combination therapy (WBRT with stereotactic radiation and/or surgery) 
$\geq 14$ days prior to randomization or single agent modality (WBRT, stereotactic radiation, or surgical resection, if combination therapy is contraindicated) $\geq 7$ days prior to randomization. Patients must have sufficiently recovered from local BCBM treatment with stable signs and symptoms of BM per the investigator. For patients who have received WBRT, or stereotactic radiation and/or surgical resection $\geq 28$ days prior to randomization, the signs or symptoms of $\mathrm{BM}$ must be stable $\geq 28$ days prior to randomization. An eligible patient may therefore have received definitive local therapy within the 28-day screening period as long as clinically stable. Corticosteroids may be used as long as patients are on a stable or decreasing dose for $\geq 7$ days prior to randomization. Prior systemic therapy (administered in the neoadjuvant, adjuvant and/or metastatic setting) must have included an anthracycline, a taxane and capecitabine (prior anthracycline may be omitted if not medically appropriate or contraindicated for the patient) and the last dose of chemotherapy must have been administered within 6 months of the date of randomization. Depending on receptor status, specific mandates for previous treatment with cytotoxic agents, hormonal therapy and/or HER2-targeted agents are required for eligibility (Supplementary Table 1). Of note, HER2-targeted therapy must be stopped in any patient with HER2-positive disease $\geq 14$ days prior to randomization. Patients may have received an unlimited number of prior therapies for advanced breast cancer; minimum therapy is listed in Supplementary Table 1. Patients must have an ECOG performance status of 0 or 1 with demonstration of adequate organ function. All anticancer- and radiation therapy- related toxicities must be completely resolved or downgraded to grade 1 or less according to National Cancer Institute Common Terminology Criteria for Adverse Events (NCI-CTCAE) version 4.03, except for diarrhea, which must be completely resolved to baseline without supportive antidiarrheal medications. Stable sensory neuropathy must be resolved to grade $\leq 2$, and alopecia can be any grade.

\section{Planned sample size \& study period}

ATTAIN is expected to be conducted in 11 countries in three regions. Recruitment is ongoing with a planned accrual of up to 220 patients. The interim analysis for OS (in which the final number of projected events will be estimated) is anticipated to occur in the second half of 2019. Depending on the number of OS events required for the final analysis, the timing of the final analysis is currently expected to occur sometime between mid-2020 to early 2021.

\section{Efficacy \& safety evaluation}

Clinical laboratory tests will be performed by a central laboratory and used to determine eligibility and treatment decisions; in situations where central laboratory results are not available, the Medical Monitor may approve use of a local laboratory. Demographics and baseline disease characteristics are collected at the screening visit, together with a physical examination, vital signs, bodyweight, medical history, complete blood count (CBC) with differential, serum chemistry, urinalysis, prothrombin time, tumor receptor status and a detailed history of prior cancer therapies, surgeries and radiotherapy. A serum pregnancy test is required at screening, $\leq 5$ days prior to each cycle and at the end-of-treatment (EOT) visit.

Tumor assessments will be performed at screening, every 8 weeks ( \pm 7 days) through week 24 and every 12 weeks ( \pm 7 days) thereafter until documented disease progression (PD) or death. To ensure that both groups are assessed for progression in a similar manner, tumor assessments will be obtained at this interval, regardless of delays in chemotherapy due to toxicity. The same method of assessment and technique for acquisition of tumor assessment data must be used to characterize each identified and reported lesion at each measurement. All patients must have tumor assessments performed at the participating study center or a radiology facility associated with the site. Tumor measurements will be evaluated locally and centrally per RECIST and RANO-BM criteria for non-CNS and CNS disease, respectively. All tumor imaging (head, chest, abdomen and other as appropriate) and digital photography must be forwarded to a central imaging facility to permit blinded independent review (local assessment will be used for patient management and continue until PD).

The safety assessments include CBC with differential, serum chemistry, vital signs and symptom-directed physical exam $\leq 5$ days prior to each cycle and EOT. Treatment decisions require results from the following tests: hemoglobin, absolute neutrophil count (ANC) and platelets. In addition, patients randomized to EP require testing for electrolytes (bicarbonate $/ \mathrm{CO}_{2}$ ), calcium, chloride, potassium, sodium and serum creatinine. Additional requirements for TPC patients should follow institutional guidelines. Dose delays, reductions and discontinuations due to adverse events (AEs) are defined in the protocol for EP and made according to the prescribing information or local practice guidelines for TPC. Patients randomized to EP will receive loperamide for treatment of diarrhea 
and/or prophylaxis (at the discretion of the investigator and/or patient preference in the absence of constipation starting with cycle 2 to mitigate the risk for late-onset diarrhea). Use of growth factor support in the setting of neutropenia is permitted and must follow American Society of Clinical Oncology (ASCO) guidelines, European Society for Medical Oncology (ESMO) guidelines, or standard of care at the local institution. Other supportive care and permitted and/or prohibited concomitant treatments are listed in Supplementary Table 2.

All patients will complete HRQoL assessments, consisting of the European Organisation for Research and Treatment of Cancer (EORTC; version 3.0) Quality of Life Core 30 (QLQ-C30) with the brain neoplasms 20question subscale (BN-20) questionnaire, the EuroQol 5D (EQ 5D 5L $\mathrm{L}^{\mathrm{TM}}$ ) questionnaire, and the Brief Fatigue Inventory (BFI) on day 1 prior to infusion for each cycle and at EOT. If possible, patients should complete the questionnaire in the same setting each time. For those patients who consent, serial blood samples for biomarker analysis will be obtained prior to cycle 1 dosing, prior to cycle 2 dosing and at the EOT visit; analysis will consist of cell-free circulating tumor DNA using next-generation sequencing to correlate with efficacy. Additionally, patients randomized to EP will be tested for the following: presence of UGT1A1 gene alteration (cycle 1, day 1); PK analyses of EP, irinotecan and its metabolites SN38, SN38-glucuronide and 7-ethyl-10-(4- $N$-(5-aminopentanoic acid)-1-piperidine) carbonyloxycamptothecin (APC; prior to each dose); and, for those patients who consent, PEG-antibody measurements in blood (prior to cycle 1, prior to cycle 2 and EOT).

After discontinuation of therapy, all patients except those who withdraw consent will be followed (by contact via phone, clinic visit, or chart views) at least every 12 weeks ( \pm 2 weeks) until death. If allowed by country regulatory authorities and consented to by the patient, study personnel may use public records to check for mortality for any patient considered lost to follow-up and for patients who withdraw consent for follow-up contact. Data will be collected on subsequent anticancer therapies in both treatment groups from the time patients come off the study treatment until the time of primary data analysis for OS.

\section{Statistical considerations}

The study uses an adaptive design powered for detecting superiority of EP compared with TPC for the primary efficacy end point of OS. The number of death events needed to provide $80 \%$ conditional power for the final analysis will be determined at an interim analysis when approximately 82 death events are available using the promising zone adaptive method [64]. Two-sided $\alpha$ of 0.001 will be used to test efficacy at the interim analysis. The minimum and maximum number of death events for the final analysis will be 106 and 191, respectively. The maximum (191) death events will be sufficient to demonstrate statistical significance for any observed hazard ratio of 0.75 or better, which corresponds to a median difference of approximately 2 months if the median OS for TPC is 6 months and the proportional hazard model assumption is approximately met. If OS does not reach statistical significance, event size needed for the final OS analysis will be determined based on conditional power per the adaptation rules described in the Data Monitoring Committee (DMC) charter.

The main efficacy analyses for the primary and secondary end points (except ORR and DoR) will be performed in the intention-to-treat (ITT) population (i.e., all randomized patients). The secondary end point analyses of ORR and DoR will utilize the Response Evaluable Population, which consists of all patients who are randomized in the study with measurable disease in the periphery by RECIST at baseline (as determined by the investigator). Safety analyses will be conducted in all patients who are randomized and receive at least one dose (or partial dose) of study drug (EP or TPC). The PK and biomarker populations will be those patients with sufficient data to permit analysis.

The primary analysis of OS will be the Cui, Hung and Wang (CHW) test with prespecified weights [65] to ensure type I error control and the conventional test with equal weights for every patient will be conducted as a sensitivity analysis. The hazard ratio (EP/TPC) and its 95\% CI will be calculated using a Cox regression model adjusting for geographic region, tumor receptor status and ECOG. The two-sided significance level for superiority at final analysis of OS will be 0.0499 . If more than $10 \%$ of the study population (i.e., $>22$ patients) received local treatment for CNS lesions (SRS, WBRT or surgery) during the study, the proportion of patients who received treatment for CNS lesions will be compared between treatment groups using Fisher's exact test. The impact of treatment for CNS lesions on OS will be evaluated using a Cox regression model comparing patients who received treatment for CNS lesions with those who did not. Additional secondary efficacy analyses will be undertaken, including (1) the difference in survival outcome depending on prior radiotherapy (SRS vs WBRT); (2) the extent of tumor burden at study entry (two or fewer vs three or more sites of disease); (3) the impact of liver metastases; and (4) the impact of removal of all patients selecting ixabepilone as the TPC agent. 
Secondary end points (PFS, ORR, CBR and DoR) will be assessed by the investigator as well as by independent central review. Analysis for secondary end points will not include any adjustment for multiplicity. Statistical tests will be two-sided with a type I error rate of 0.05. Progression-free survival (PFS-outside the CNS, PFS-BM and PFS-overall) will be compared with a two-sided, log-rank test with the same stratification factors that were used for randomization. Two sensitivity analyses of PFS (outside the CNS) will be performed based on the censoring criteria with considerations of informative censoring as described in the statistical analysis plan. Kaplan-Meier median PFS times and their 95\% CI, as well as PFS curves, will be summarized by treatment group. The hazard ratio (EP/TPC) and its 95\% CI will be calculated using a Cox regression model adjusting for geographic region, tumor receptor status, and baseline ECOG status. The analysis of ORR will be performed using Fisher's exact test. Clinical benefit rate (defined as $\mathrm{CR}, \mathrm{PR}$ or $\mathrm{SD} \geq 4$ months to reflect shorter life expectancy of the BM population) will be compared between the treatment groups using a Cochran Mantel-Haenszel test stratified by the randomization factors. Clopper Pearson exact two-sided $95 \%$ confidence limits will be calculated in determining the proportion of patients with ORR and CBR in each treatment group. Additionally, the magnitude of clinical benefit of EP will be assessed by the ESMO-magnitude of clinical benefit scale (ESMO-MCBS, version 1.0); this information will be summarized and applied to comparative outcome studies evaluating the relative benefit of treatments using outcomes of survival, quality of life, surrogate outcomes for survival, or treatment toxicity in solid cancers. Kaplan-Meier median DoR curves will be summarized by treatment group. DoR will be calculated based on the central imaging facility assessment of response and progression, as well as the investigator's assessment of response and progression. Incidence and frequency of WBRT, as well as time to WBRT, will be compared and summarized by treatment group. Summary statistics of absolute scores at each assessment point and changes from baseline will be calculated by treatment group for HRQoL measures. Changes from baseline will be compared between treatment groups using Repeated Measures Linear Mixed Effects and Generalized Linear Mixed Models Analyses. The safety analysis will be summarized by treatment group (EP and TPC) and further tabulated for each TPC drug. Additional safety analyses will be undertaken to investigate: (1) diarrhea, neutropenia, neuropathy and hypersensitivity reactions; (2) pulmonary toxicity including pneumonitis and interstitial lung disease; and, (3) the impact of prophylactic loperamide on the management of diarrhea. All additional analyses (laboratory, PK, UGT1A1 mutation, PEG-antibodies and biomarker) will be summarized with descriptive statistics; exploratory analyses will be undertaken to correlate selected biomarkers as well as the appearance of PEG-antibodies with ORR, PFS and OS.

\section{Conclusion}

There remains a critical need for therapeutic interventions that prolong patient survival and maintain or improve quality of life of patients with BCBM, in particular, using agents that are effective against both CNS and systemic disease. The lack of consensus regarding treatment and the lack of robust data supporting current clinical practices demonstrate the need for more controlled trials in this setting. Etirinotecan pegol (EP) is a next-generation, long-acting topoisomerase- 1 inhibitor engineered to penetrate into the brain, provide sustained anti-tumor activity (systemically and within the CNS) and reduce systemic toxicities. The ATTAIN trial will compare the survival of patients receiving EP to that of standard of care agents in the treatment of patients with $\mathrm{MBC}$ and stable BM. The trial is a registration trial, which is currently actively recruiting eligible patients. If ATTAIN meets its primary end point, EP treatment will establish a new and much needed therapeutic option for patients with BCBM.

\section{Supplementary data}

To view the supplementary data that accompany this paper please visit the journal website. An infographic also accompanies this paper at the end of the references section. To download the infographic that accompanies this paper, please visit the journal website at: www.futuremedicine.com/doi/full/10.2217/fon-2019-0180

\section{Financial \& competing interests disclosure}

D Tripathy receives institutional research funding from Novartis, consulting fees from Pfizer and Novartis, and serves in an unpaid member of the Scientific Advisory Board for Puma Biotechnology.

SM Tolaney receives institutional research funding from Novartis, Genentech, Eli Lilly, Pfizer, Merck, Exelixis, Eisai, Bristol Meyers Squibb, AstraZeneca, Cyclacel, Immunomedics, Odenate, and Nektar. Tolaney has served as an advisor/consultant to Novartis, Eli Lilly, Pfizer, Merck, AstraZeneca, Eisai, Puma, Genentech, Immunomedics, Nektar, Tesaro, and Nanostring. 
AD Seidman receives institutional research funding from Novartis, Odonate, and Nektar. Seidman has served as an advisor/consultant to Genomic Health, Novartis, Eli Lilly, Pfizer, AstraZeneca, Eisai, Puma, and Genentech.

C Anders receives researching funding from Novartis, Merrimack, PUMA, Lily, Merck, Seattle Genetics, Nektar, Tesaro, and G1Therapeutics. Anders has served in an uncompensated advisory role for Merck (Start 6/2018-6/2023), Novartis, Merrimack, Eli Lilly, Nektar, and Seattle Genetics as well as a compensated advisory role for PUMA (9/2018-9/2019), Genentech (1/2019-present), Eisai (1/2019-present), and IPSEN (2/2019-present). She also received royalties from UpToDate, Jones and Bartlett.

$\mathrm{N}$ Ibrahim receives research funding from Nektar Therapeutics. He also serves on the advisory board for Ipsen and Immunogenetics.

HS Rugo receives research support for clinical trials through the University of California from: Pfizer, Merck, Novartis, Lilly, Genentech, OBI, Odonate, Daiichi, Eisai, Seattle Genetics and Macrogenics, and Immunomedics. She has also received travel support from: Daiichi, Mylan, Pfizer, Amgen, Merck and Puma.

C Twelves has served as a consultant/advisor and received honoraria or travel funding from Roche, Daiichi Sankyo, Eisai, Diagenode, Amcure and Pfizer over the past 5 years.

V Diéras has received honoraria as a consultant and/or speaker for Roche/Genentech, Novartis, Eli Lilly, Pfizer, Eisai, Nektar, Astellas, AbbVie, MSD, Tesaro, Daiichi Sankyo, Odonate, Seattle Genetics, and AstraZeneca. Diéras has also received funding for travel expenses from Roche, Novartis, Pfizer, GSK, Eisai, and AstraZeneca.

V Müeller has received honoraria directly from Amgen, AstraZeneca, Celgene, Daiichi-Sankyo, Eisai, Pfizer, Novartis, Roche, Teva, and Janssen-Cilag. Müeller has received payment for acting as a consult or advisor for Hexal, Roche, Pfizer, Amgen, Daiichi-Sankyo, Nektar, and Eisai. He has also received research funding from Roche, Novartis, Seattle Genetics, and Pfizer.

MA Tagliaferri is CMO at Nektar and has ownership interest (including stock, patents, etc.) in the same.

AL Hannah is a consultant to Nektar Therapeutics.

J Cortes has served as a consultant/advisor for Roche, Celgene, Cellestia, AstraZeneca, Biothera Pharmaceutical, Merus, Seattle Genetics, Daiichi Sankyo, Erytech, and Athenex. He has received honoraria from Roche, Novartis, Celgene, Eisai, Pfizer, Samsung. Cortes has also received institutional research funding from Roche, Ariad pharmaceuticals, AstraZeneca, Baxalta GMBH/Servier Affaires, Bayer healthcare, Eisai, F Hoffman-La Roche, Guardanth health, Merck Sharp \& Dohme, Pfizer, Piqur Therapeutics, Puma C, and Queen Mary University of London. He also owns stock in MedSIR.

The trial is sponsored by Nektar Therapeutics. This research was supported by the National Institute for Health Research Leeds Clinical Research Facility. The authors have no other relevant affiliations or financial involvement with any organization or entity in conflict with the subject matter or materials discussed in the manuscript apart from those disclosed.

Medical writing assistance was provided by Phillips Gilmore Oncology Communications (PA, USA), funded by Nektar Therapeutics.

\section{Open access}

This work is licensed under the Attribution-NonCommercial-NoDerivatives 4.0 Unported License. To view a copy of this license, visit http://creativecommons.org/licenses/by-nc-nd/4.0/ 
Breast cancer brain metastases

- Breast cancer brain metastases (BCBM) remain a challenging consequence of advanced disease with cumulative incidence rates approaching or exceeding $35-50 \%$, particularly in high-risk populations.

- The development of BCBM is associated with a poor prognosis and detrimental effects on quality of life.

- Treatment options for patients with BCBM following prior local surgery and/or radiotherapy remain limited with existing chemotherapy hindered by poor penetration and distribution into the CNS.

- As deemed a critical unmet medical need, there is increasing optimism and interest in exploring novel treatments for this challenging clinical problem.

Etirinotecan pegol (also called NKTR-102)

- Etirinotecan pegol (EP) is a next-generation, long-acting topoisomerase-1 inhibitor engineered to produce sustained exposure to irinotecan and its active metabolite SN38.

- A mouse xenograft model of BCBM demonstrated preferential accumulation of EP in brain tumors, a decrease in both tumor burden and lesion size and extended survival times compared with conventional irinotecan.

- Etirinotecan pegol avoids P-glycoprotein and BCRP/ABCG2-mediated efflux, providing a potential added benefit for uptake in brain lesions.

- In a prespecified subgroup analysis of the randomized Phase III BEACON trial, EP prolonged survival in patients with breast cancer and stable brain metastases (BM) compared with commonly used drugs for the treatment of advanced breast cancer.

The ATTAIN trial

- ATTAIN is a randomized Phase III trial of etirinotecan pegol (EP) versus treatment of physician's choice (TPC) in patients with MBC who have stable BM.

- Primary end point is overall survival.

- Key secondary end points include objective response rate, clinical benefit rate, duration of response and progression-free survival (assessed by local investigator and independent central review); additionally, review of CNS lesions will be performed using RANO-BM guidelines [63].

- Other key secondary end points include time to CNS disease progression in patients with CNS lesions present at study entry, time to CNS recurrence in patients without CNS lesions present at study entry, toxicity, magnitude of clinical benefit assessed by the European Society for Medical Oncology magnitude of clinical benefit scale (ESMO-MCBS, v1.0), incidence and cumulative frequency of WBRT, time-to-WBRT in those patients who received WBRT and health-related quality of life.

- Eligibility includes patients with historically-confirmed breast cancer, either measurable or nonmeasurable metastatic disease, stable treated (whole-brain or stereotactic radiation and/or surgical resection) brain metastases, prior treatment with an anthracycline (unless not medically appropriate or contraindicated), a taxane and capecitabine administered in the neoadjuvant, adjuvant and/or metastatic setting (last dose of chemotherapy must have been administered within 6 months of randomization), and an Eastern Cooperative Oncology Group (ECOG) performance status of 0 or 1.

- Depending on receptor status, 1 or 2 prior cytotoxic regimens are required prior to enrollment in this trial; hormonal and/or HER2-targeted agents may be required (minimum requirements for number of prior regimens may be found in Supplementary Table 1).

- This study will randomize patients $1: 1$ to either etirinotecan pegol $145 \mathrm{mg} / \mathrm{m}^{2}$ as a 90 -minute intravenous (iv.) infusion on day 1 of a 21-day cycle or to TPC (administered per standard of care) and stratified based on geographic region, tumor receptor status and ECOG status.

- Patients randomized to TPC will receive single-agent iv. chemotherapy, limited to choice of one of the following seven agents: eribulin, ixabepilone, vinorelbine, gemcitabine, paclitaxel, docetaxel or nab-paclitaxel (doublet therapy, including trastuzumab-based doublets) are not permitted.

- The proposed sample size of 220 patients provides $80 \%$ power to detect a HR of 0.75 or better, corresponding to a 2-month increase in survival favoring etirinotecan pegol at a two-sided significance level of 0.05.

- This trial is expected to be conducted in 11 countries in three regions; for participating trial sites, please visit: https://clinicaltrials.gov and search NCT02915744. 


\section{References}

Papers of special note have been highlighted as: $\bullet$ of interest; $\bullet \bullet$ of considerable interest

1. Martin AM, Cagney DN, Catalano PJ et al. Brain metastases in newly diagnosed breast cancer. A population-based study. JAMA Oncol. 3(8), 1069-1077 (2017).

2. Rostami R, Mittal S, Rostami P, Tavassoli F, Jabbari B. Brain metastasis in breast cancer: a comprehensive literature review. J. Neurooncol. 127(3), 407-414 (2016).

3. Gil-Gil MJ, Martinez-Garcia M, Sierra A et al. Breast cancer brain metastases: a review of the literature and a current multidisciplinary management guideline. Clin. Transl. Oncol. 16, 436-446 (2014).

4. Tabouret E, Chinot O, Metellus P, Tallet A, Viens P, Goncalves A. Recent trends in epidemiology of brain metastases: an overview. Anticancer Res. 32, 4655-4662 (2012).

- Review of recent trends in brain metastases highlighting epidemiologic characteristics, rationalized use of surgical resection, radiosurgery and whole-brain radiotherapy, and progress in the development of systemic treatments.

5. Witzel I, Oliveira-Ferrer L, Pantel K, Muller V, Wikman H. Breast cancer brain metastases: biology and new clinical perspectives. Breast Cancer Res. 18(1), 8 (2016).

6. Zagar TM, Van Swearingen AE, Kaidar-Person O, Ewend MG, Anders CK. Multidisciplinary management of breast cancer brain metastases. Oncology (Williston Park) 30(10), 923-933 (2016).

7. Leone JP, Lee AV, Brufsky AM. Prognostic factors and survival of patients with brain metastasis from breast cancer who underwent craniotomy. Cancer Med. 4(7), 989-994 (2015).

8. Soni A, Ren Z, Hameed O et al. Breast cancer subtypes predispose the site of distant metastases. Am. J. Clin. Pathol. 143(4), 471-478 (2015).

9. Aversa C, Rossi V, Geuna E et al. Metastatic breast cancer subtypes and central nervous system metastases. Breast 23(5), 623-628 (2014).

10. Lim E, Lin NU. Updates on the management of breast cancer brain metastases. Oncology (Williston Park) 28(7), 572-578 (2014).

11. Anderson A, Choy C, Neman J, Duenas MJ, Jandial R, Somlo G. Metastatic breast cancer to the brain: a clinical primer for translational investigation. In: Madame Curie Bioscience Database. Landes Bioscience, Austin, TX, USA (2013).

12. Lim E, Lin NU. New insights and emerging therapies for breast cancer brain metastases. Oncology (Williston Park) 26, 652-659, 663 (2012).

13. Lin NU, Vanderplas A, Hughes $\mathrm{M}$ et al. Clinicopathological features, patterns of recurrence, and survival among women with triple-negative breast cancer in the National Comprehensive Cancer Network. Cancer 118(22), 5463-5472 (2012).

14. Kennecke H, Yerushalmi R, Woods R et al. Metastatic behavior of breast cancer subtypes. J. Clin. Oncol. 28(20), 3271-3277 (2010).

15. Niwińska A, Murawska M, Pogoda K. Breast cancer brain metastases: differences in survival depending on biological subtype, RPA RTOG prognostic class and systemic treatment after whole-brain radiotherapy (WBRT). Ann. Oncol. 21, 942-948 (2010).

16. Venur VA, Leone JP. Targeted therapies for brain metastases from breast cancer. Int. J. Mol. Sci. 17(9), 1543 (2016).

17. Moja L, Tagliabue L, Balduzzi S et al. Trastuzumab containing regimens for early breast cancer. Cochrane Database Syst. Rev. 4, CD006243 (2012).

18. Khuntia D. Contemporary review of the management of brain metastasis with radiation. Adv. Neurosci. 2015, 1-13 (2015).

19. Leone JP, Leone BA. Breast cancer brain metastases: the last frontier. Exp. Hematol. Oncol. 4, 33 (2015).

20. Mehta M. The controversy surrounding the use of whole-brain radiotherapy in brain metastases patients. Neuro. Oncol. 17(7), 919-923 (2015).

21. Sahgal A. Point/counterpoint: stereotactic radiosurgery without whole-brain radiation for patients with a limited number of brain metastases: the current standard? Neuro. Oncol. 17(7), 916-918 (2015).

22. Lombardi G, Di Stefano AL, Farina P, Zagonel V, Tabouret E. Systemic treatments for brain metastases from breast cancer, non-small cell lung cancer, melanoma and renal cell carcinoma: an overview of the literature. Cancer Treat. Rev. 40(8), 951-959 (2014).

23. Tsao M, Xu W, Sahgal A. A meta-analysis evaluating stereotactic radiosurgery, whole-brain radiotherapy, or both for patients presenting with a limited number of brain metastases. Cancer 118, 2486-2493 (2012).

24. Sperduto PW, Kased N, Roberge D et al. Summary report on the Graded Prognostic Assessment: an accurate and facile diagnosis-specific tool to estimate survival for patients with brain metastases. J. Clin. Oncol. 30(4), 419-425 (2012).

25. Sperduto PW, Kased N, Roberge D et al. Effect of tumor subtype on survival and the graded prognostic assessment (GPA) for patients with breast cancer and brain metastases. Int. J. Radiat. Oncol. Biol. Phys. 82(5), 2111-2117 (2012).

-. The data presented confirmed the effect of tumor subtype on survival and demonstrated that breast-GPA offers significantly more predictive power than tumor subtype alone. The proposed breast-GPA index has the potential to alter clinical management of patients with breast cancer and brain metastases through 1) individualized clinical decision making; 2) comparing trials; 3) re-analyzing prior trials; 4) stratifying patients enrolled in future prospective trials; 5) guiding clinical trial development, and; 6) designing treatment guidelines. 
26. Niwińska A, Murawska M, Pogoda K. Breast cancer subtypes and response to systemic treatment after whole-brain radiotherapy in patients with brain metastases. Cancer 116(18), 4238-4247 (2010).

27. Brown PD, Jaeckle $\mathrm{K}$, Ballman KV et al. Effect of radiosurgery alone vs radiosurgery with whole brain radiation therapy on cognitive function in patients with 1 to 3 brain metastases: a randomized clinical trial. JAMA 316(4), 401-409 (2016).

28. Sahgal A, Aoyama H, Kocher M et al. Phase 3 trials of stereotactic radiosurgery with or without whole-brain radiation therapy for 1 to 4 brain metastases: individual patient data meta-analysis. Int. J. Radiat. Oncol. Biol. Phys. 91(4), 710-717 (2015).

29. Soon YY, Tham IW, Lim KH, Koh WY, Lu JJ. Surgery or radiosurgery plus whole brain radiotherapy versus surgery or radiosurgery alone for brain metastases. Cochrane Database Syst. Rev. 3, CD009454 (2014).

30. Tallet AV, Azria D, Barlesi F et al. Neurocognitive function impairment after whole brain radiotherapy for brain metastases: actual assessment. Radiat. Oncol. 7, 77 (2012).

31. Kocher M, Soffietti R, Abacioglu U et al. Adjuvant whole-brain radiotherapy versus observation after radiosurgery or surgical resection of one to three cerebral metastases: results of the EORTC 22952-26001 study. J. Clin. Oncol. 29(2), 134-141 (2011).

32. Agarwal S, Hartz AMS, Elmquist WF, Bauer B. Breast cancer resistance protein and p-glycoprotein in brain cancer: two gatekeepers team up. Curr. Pharm. Des. 17(26), 2793-2802 (2011).

33. Lockman PR, Mittapalli RK, Taskar KS et al. Heterogeneous blood-tumor barrier permeability determines drug efficacy in experimental brain metastases of breast cancer. Clin. Cancer Res. 16(23), 5664-5678 (2010).

34. Kalsi R, Feigenberg S, Kwok Y et al. Brain metastasis and response to ado-trastuzumab emtansine: a case report and literature review. Clin. Breast Cancer 15(2), e163-e166 (2015).

35. Bartsch R, Berghoff AS, Vogl U et al. Activity of T-DM1 in Her2-positive breast cancer metastases. Clin. Exp. Metastasis 32(7), 729-737 (2015).

36. Keith KC, Lee Y, Ewend MG et al. Activity of trastuzumab-emtansine (TDM1) in HER2-positive breast cancer brain metastases: a case series. Cancer Treat. Commun. 7, 43-46 (2016).

37. Ramakrishna N, Temin S, Chandarlapaty S et al. Recommendations on disease management for patients with advanced human epidermal growth factor receptor 2 positive breast cancer and brain metastases: American Society of Clinical Oncology clinical practice guideline. J. Clin. Oncol. 32(19), 2100-2108 (2014).

38. Lin NU. Breast cancer brain metastases: new directions in systemic therapy. Ecancermedicalscience 7, 307 (2013).

39. Arslan C, Dizdar O, Altundaq K. Chemotherapy and biological treatment options in breast cancer patients with brain metastasis: an update. Expert Opin. Pharmacother. 15(12), 1643-1558 (2014).

40. Cortés J, Dieras V, Ro J et al. Afatinib alone or afatinib plus vinorelbine versus investigator's choice of treatment for HER2-positive breast cancer with progressive brain metastases after trastuzumab, lapatinib, or both (LUX-breast 3): a randomised, open-label, multicentre, Phase 2 trial. Lancet Oncol. 16(16), 1700-1710 (2015).

41. Peereboom DM, Murphy C, Ahluwalia MS et al. Phase II trial of patupilone in patients with brain metastases from breast cancer. Neuro. Oncol. 16(4), 579-583 (2014).

42. Freedman RA, Bullitt E, Sun L et al. A Phase II study of sagopilone (ZK 219477; ZK-EPO) in patients with breast cancer and brain metastases. Clin. Breast Cancer 11(6), 376-383 (2011).

43. Hamilton E, Borges V, Conlin A et al. Efficacy results of a Phase $1 \mathrm{~b}$ study of ONT-380, an oral HER2-specific inhibitor, in combination with capecitabine and trastuzumab in HER2+ metastatic breast cancer, including patients with brain metastases. Presented at: 39th Annual CTRC-AACR San Antonio Breast Cancer Symposium, San Antonio, TX, USA, 6-10 December 2016.

44. Borges VF, Ferrario C, Aucoin N et al. Efficacy results of a Phase $1 \mathrm{~b}$ study of ONT-380, a CNS-penetrant TKI, in combination with T-DM1 in HER2+ metastatic breast cancer, including patients with brain metastases. Presented at: $52^{\text {nd }}$ Annual Meeting of the American Society of Clinical Oncology, Chicago, IL, USA, 3-9 June 2016.

45. Twelves C, Jove M, Gombos A, Awada A. Cytotoxic chemotherapy: still the mainstay of clinical practice for all subtypes metastatic breast cancer. Crit. Rev. Oncol. Hematol. 100, 74-87 (2016).

46. Ayoub JP, Verma Sh, Verma S. Advances in the management of metastatic breast cancer: options beyond first-line chemotherapy. Curr Oncol. 19(2), 91-105 (2012).

47. Marquette C, Nabell L. Chemotherapy-resistant metastatic breast cancer. Curr. Treat. Options Oncol. 13(2), 263-275 (2012).

48. Hoch U, Staschen C-M, Johnson R, Eldon MA. Nonclinical pharmacokinetics and activity of etirinotecan pegol (NKTR-102), a long-acting topoisomerase 1 inhibitor, in multiple cancer models. Cancer Chemother. Pharmacol. 74(6), 1125-1137 (2014).

49. Sy SK, Sweeney TD, Ji C, Hoch U, Eldon MA. Etirinotecan pegol administration is associated with lower incidences of neutropenia compared to irinotecan administration. Cancer Chemother. Pharmacol. 79(1), 57-67 (2017).

50. Camptosar [Package Insert]. Pfizer, NY, USA 2016. http://labeling.pfizer.com/ShowLabeling.aspx?format=PDF\&id=533

51. Jameson GS, Hamm JT, Weiss GJ et al. Multicenter, Phase I, dose-escalation study to assess the safety, tolerability, and pharmacokinetics of etirinotecan pegol in patients with refractory solid tumors. Clin. Cancer Res. 19(1), 268-278 (2013). 
- First-in-human study of etirinotecan pegol that demonstrated antitumor activity in a broad spectrum of cancers, including breast cancer. Importantly, cholinergic diarrhea associated with conventional irinotecan was not seen with etirinotecan pegol in the dosing regimens studied.

52. Yin H, Liao L, Fang J. Enhanced permeability and retention (EPR) effect based tumor targeting: the concept, application and prospect. JSM Clin. Oncol. Res. 2(1), 1010 (2014).

53. Greish K. Enhanced permeability and retention (EPR) effect for anticancer nanomedicine drug targeting. Methods Mol. Biol. 624, 25-37 (2010).

54. Shah N, Mohammad AS, Adkins CE et al. Etirinotecan pegol: survival advantage over standard of care drugs in a model of brain metastases of breast cancer. Presented at: 39th Annual CTRC-AACR San Antonio Breast Cancer Symposium, TX, USA, 6-10 December 2016.

55. Adkins CE, Nounou MI, Hye T et al. NKTR-102 Efficacy versus irinotecan in a mouse model of brain metastases of breast cancer. $B M C$ Cancer 15, 685 (2015).

-. Data reported demonstrated the efficacy of etirinotecan pegol in an experimental mouse model of breast cancer brain metastases (BCBM). The authors concluded that the efficacy observed correlated with the ability of etirinotecan to cross the blood-tumor barrier, leading to preferential accumulation and retention in brain tumor, followed by sustained efficacious concentrations of the active metabolite, SN38. The data provide a strong rationale for the clinical use of etirinotecan pegol in patients diagnosed with BCBM.

56. Nagpal S, Recht CK, Bertrand S et al. Phase II pilot study of single-agent etirinotecan pegol (NKTR-102) in bevacizumab-resistant high grade glioma. J. Neurooncol. 123(2), 277-282 (2015).

-. This Phase II pilot trial of etirinotecan pegol in the treatment of heavily-pretreated (median of three prior therapies), bevacizumab-refractory, high-grade glioma, which produced three partial responses in a small cohort of patients, established foundation for further clinical investigation of the drug in brain tumors and brain metastases from SN38-sensitive primary cancers.

57. Anders C, Deal AM, Abramson V et al. TBCRC 018: Phase II study of iniparib in combination with irinotecan to treat progressive triple negative breast cancer brain metastases. Breast Cancer Res Treat. 146(3), 557-566 (2014).

58. Perez EA, Awada A, O'Shaughnessy J et al. Etirinotecan pegol (NKTR-102) versus treatment of physician's choice in women with anthracycline-, taxane-, and capecitabine-treated advanced breast cancer: a randomised, open-label, multicentre, Phase 3 trial. Lancet Oncol. 16(15), 1556-1568 (2015).

-• A randomized Phase III trial of etirinotecan pegol versus treatment of physician choice in women with anthracycline-, taxane-, and capecitabine-treated advanced breast cancer that demonstrated a non-statistical 2-month survival advantage favoring etirinotecan pegol. In pre-specified subgroups with brain and liver metastases, there was a significant $\mathrm{OS}$ benefit of 5.2 months $(p<0.01)$ and 2.6 months $(p<0.002)$, respectively. Etirinotecan pegol treatment was associated with a favorable tolerability profile and improved quality of life.

59. Cortés J, Rugo HS, Awada A et al. Prolonged survival in patients with breast cancer and a history of brain metastases: results of a preplanned subgroup analysis from the randomized Phase III BEACON trial. Breast Cancer Res. Treat. 165, 329-341 (2017).

-. This preplanned subgroup analysis from the Phase III BEACON trial found that etirinotecan pegol resulted in a significant improvement in median OS (10 vs 4.8 months; HR: 0.51; $\mathrm{p}<0.001)$ compared with physician's choice chemotherapy in patients with breast cancer brain metastases. Etirinotecan pegol treatment was associated with fewer grade $\geq 3$ adverse events, which was consistent with the overall BEACON population.

60. Cortés J, Rugo HS, Twelves C et al. Safety and tolerability of etirinotecan pegol in advanced breast cancer: analysis of the randomized, Phase 3 BEACON trial. Springerplus 5(1), 1033 (2016).

61. Twelves C, Cortés J, O'Shaughnessy J et al. Health-related quality of life in patients with locally recurrent or metastatic breast cancer treated with etirinotecan pegol versus treatment of physician's choice: results from the randomised Phase III BEACON trial. Eur. J. Cancer 76, 205-215 (2017).

62. Eisenhauer EA, Therasse P, Bogaerts J et al. New response evaluation criteria in solid tumors: revised RECIST guideline (version 1.1). Eur. J. Cancer 45, 228-247 (2009).

63. Lin NU, Lee EQ, Aoyama $\mathrm{H}$ et al. on behalf of Response Assessment in Neuro-Oncology (RANO) group. Response assessment criteria for brain metastases: proposal from the RANO group. Lancet Oncol. 16(6), e270-278 (2015).

-. Proposed standard response and progression criteria for the assessment of brain metastases. These proposed criteria are integral for providing uniformity of CNS assessment across clinical trials. The Response Assessment in Neuro-Oncology Brain Metastases (RANO-BM) working group was an international, multidisciplinary effort.

64. Mehta C, Pocock SJ. Adaptive increase in sample size when interim results are promising: a practical guide with examples. Stat. Med. 30(28), 3267-3284 (2011).

65. Cui L, Hung JHM, Wang SJ. Modification of sample size in group sequential clinical trials. Biometrics 55(3), 853-857 (1999). 

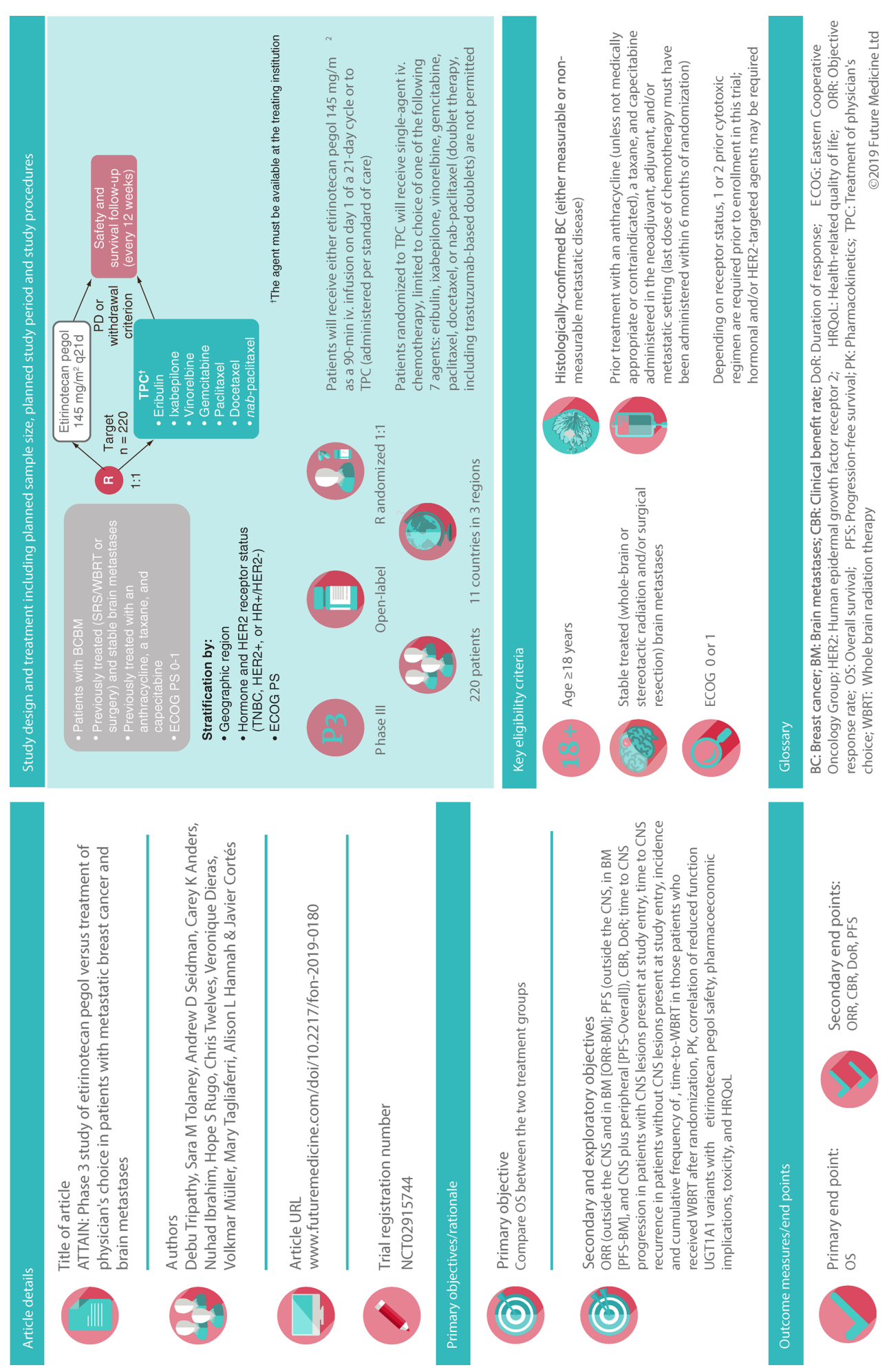